a Boer republic has been declared in the plateau region of Angola, one of the healthiest parts of tropical Africa.

THE survey of the district surrounding Aden has been com. pleted by the officers of the Survey of India Department after a very arduous campaign. Work was on several occasions almost stopped by sickness, and by the open hostility of the natives.

STIMULATED by the recent discovery of two complete mammoth carcases in the Government of Irkutsk, the St. Petersburg Academy of Sciences has commissioned Prof. Tcherski, of Irkutsk, to proceed to Yakutsk, on the Lena, and thence, accompanied by Cossacks and pack-horses, eastward to the Kolyma Valley, pushing on if possible this summer to Nizhne Kolymsk in $69^{\circ} \mathrm{N}$., returning before winter to Sachiversk on the Indigirka, a town situated on the Arctic Circle. The main object of the expedition is to study the drift geology, but collections will be made in all departments of science, including barometric observations, in order to determine the orography of this rarely visited part of Siberia.

Globus announces the formation of a new islet in the Caspian, near Baku, by upheaval. It lies three and a half miles from shore, and measures $\mathbf{I} 75$ feet by roo feet, rising about 20 feet above the water. Its surface is irregular, and composed of blackish grey and yellow hardened mud.

WITH reference to the note on p. 65 as to the discovery of a new range of mountains in Benin, it is only fair to former travellers in that region to say that the map by the Intelligence Department, although bearing no mountain shading, has marked upon it "Mt. Ara," very near the position where the range seen by Governor Carter is situated.

THE mountaineering expedition, led by $\mathrm{Mr}$. Conway, to attempt the ascent of the loftiest Himalayan summits, has been making excursions from Gilgit and mapping the Bagrot Valley, but bad weather has prevented any very important climbing from being done. A Times telegram from Calcutta conveys news of June 8 from Gilgit, from which it appears that the greatest height yet reached is $I 7,000$ feet, one night having been passed at an elevation of 15,600 feet. The party was about to set out for Nagar, en route for Askoley, by the Hissar Pass.

\section{A NEW FORM OF AIR LEYDEN.1}

$\mathrm{I}$ the title of this paper as originally offered for communica tion "Air Condenser" stood in place of "Air Leyden," but it was accompanied by a request to the Secretaries to heip me to a better designation than "Air Condenser". (with it ambiguous suggestion of an apparatus for condensing air), and I was happily answered by Lord Rayleigh with a proposal to use the word "leyden" to denote a generalized Leyden jar, which I have gladly adopted.

The apparatus to be described affords, in conjunction with a suitable electrometer, a convenient means of quickly measuring small electrostatic capacities, such as those of short lengths of cable.

The instrument is formed by two mutually insulated metallic pieces, which we shall call $\mathrm{A}$ and $\mathrm{B}$, constituting the two systems of an air condenser, or, as we shall now call it, an air leyden. The systems are composed of parallel plates, each set bound together by four long metal bolts. The two extreme plates of set $\mathrm{A}$ are circles of much thicker metal than the rest, which are all squares of thin sheet brass. The set B are all squares, the bottom of which is of much thicker metal than the others, and the plates of this system are one less in number than the plates of systen A. The four bolts binding together the plates of each system pass through well-fitted holes in the corners of the squares; and the distance from plate to plate of the same set is regulated by annular distance pieces which are carefully made to fit the bolt, and are made exactly the same in all respects. Each system is bound firmly together by screwing home nuts on the ends of the bolts, and thus the parallelism and rigidity of the entire set is secured.

The two systems are made up together, so that every plate of $B$ is between two plates of $A$, and every plate of $A$, except the two end ones, which only present one face to those of the op-

1 "On a New Form of Air Leyden, with Application to the Measurement of Small Electrostatic Capacities." Dy Lord Kelvin, P.R.S. Kead at the Royal Society on June 2 .

No. I $\delta_{3}$, vor. 46$]$ posite set, is between two plates of $B$. When the instrument is set up for use, the system B rests by means of the well-known "hole slot and plane arrangement," engraved on the under side of its bottom plate, on three glass columns which are attached to three metal screws working through the sole plate of system A. These screws can be raised or lowered at pleasure, and by means of a gauge the plates of system $\mathrm{B}$ can be adjusted to exactly midway between and parallel to the plates of system
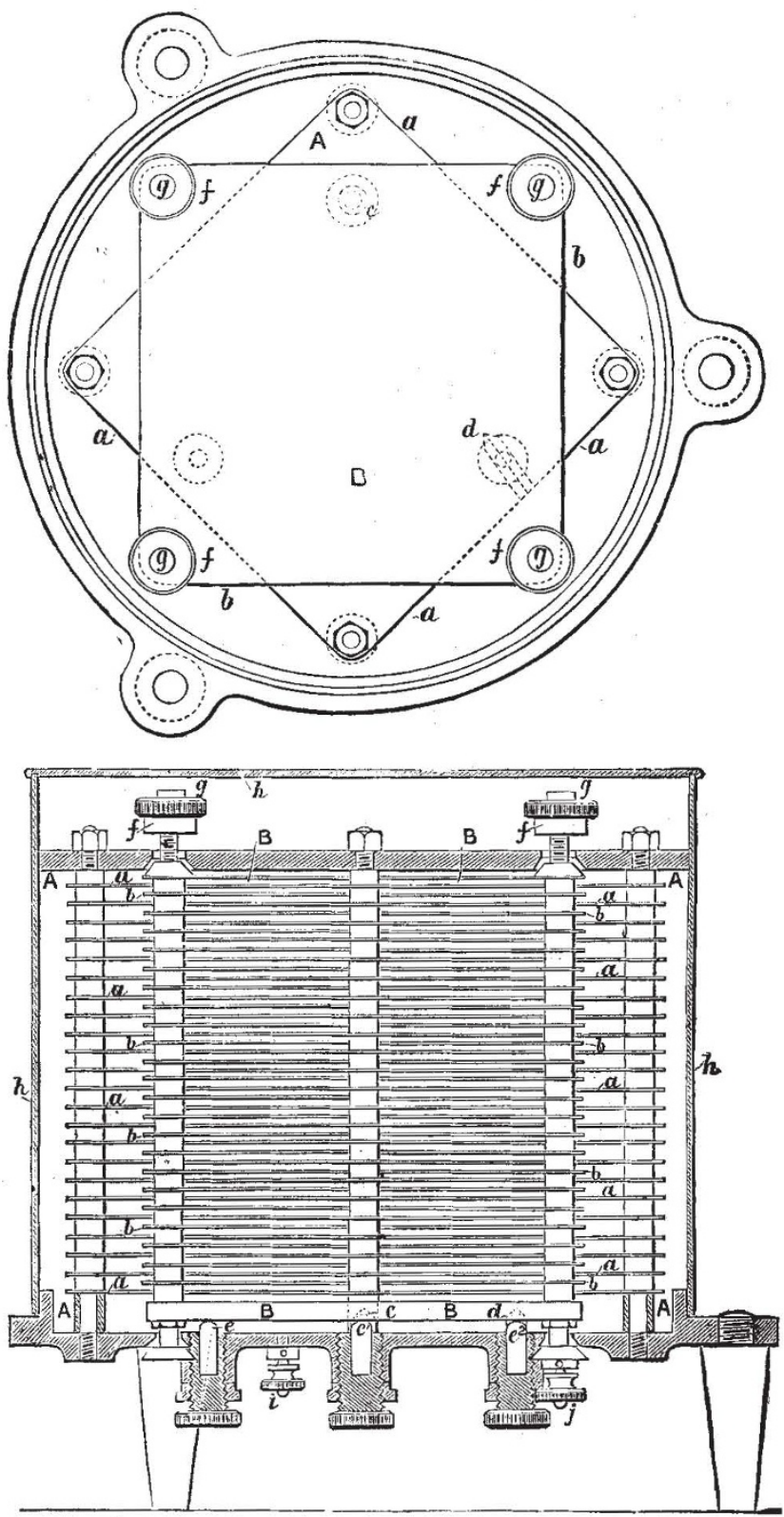

A. The complete leyden stands upon three vulcanite feet attached to the lower side of the sole plate of system A.

In order that the instrument may not be injured in carriage, an arrangement, described as follows, is provided, by which system $\mathrm{B}$ can be lifted from off the three glass columns and firmly clamped to the top and bottom plates of system A.

The bolts fixing the corners of the plates of system $B$ are made long enough to pass through wide conical holes cut in the top and bottom plates of system A, and the nuts at the top end of the bolts are also conical in form, while conical nuts are also

1 Thomsonand Tait's " Natural Philosophy," \$ $\$ 18$ example 3. 
fixed to their lower ends below the base plate of system A. Thumbscrew nuts, $f$, are placed upon the upper ends of the bolts after they pass through the holes in the top plate of system $\mathrm{A}$.

When the instrument is set up ready for use, these thumbscrews are turned up against fixed stops, $g$, so as to be well clear of the top plate of system A ; but when the instrument is packed for carriage they are screwed down against the plate until the conical nuts mentioned above are drawn up into the conical holes in the top and bottom plates of system A ; system B is thus raised off the glass pillars, and the two systems are securely locked together so as to prevent damage to the instrument.

A dust-tight cylindrical metal case, $h$, which can be easily taken off for inspection, covers the two systems, and fits on to a flange on system A. The whole instrument rests on three vulcanite legs attached to the brass plate on system $\mathrm{A}$; and two terminals are provided, one, $i$, on the base of system $\mathrm{A}$, and the other, $j$, on the end of one of the corner bolts of system $\mathrm{B}$.

The air leyden which has been thus described is used as a standard of electrostatic capacity. In the instrument actually exhibited to the Society there are twenty-two plates of the system B, twenty-three of the system $\mathrm{A}$, and therefore fortyfour octagonal air spaces between the two sets of plates. The thickness of each of these air spaces is approximately $0^{\prime} 3$ or of a centimetre. The side of each square is $10.13 \mathrm{~cm}$., and therefore the area of each octagonal air space is $85.1 \mathrm{sq} . \mathrm{cm}$. The capacity of the whole leyden is therefore approximately $44 \times 85 \cdot 1 /(4 \pi \times \cdot 287)$, or $1038 \mathrm{~cm}$. in electrostatic measure. This is only an approximate estimate, founded on a not minutely accurate measurement of dimensions, and not corrected for the addition of capacity, due to the edges and projecting angles of the squares and the metal cover. I hope to have the capacity determined with great accuracy by comparison with Mr. Glazebrook's standards in Cambridge.

To explain its use in connection with an idiostatic electrometer for the direct measurement of the capacity of any insulated conductor, I shall suppose, for example, this insulated conductor to be the insulated wire of a short length of submarine cable core, or of telephone, or telegraph, or electric light cable, sunk under water, except a projecting portion to allow external connection to be made with the insulated wire.

The electrometer which I find most convenient is my "multi. cellular voltmeter," rendered practically dead-beat by a vane under oil hung on the lower end of the long stem carrying the electric "needles" (or movable plates). In the multicellular voltmeter used in the experimental illustration before the Royal Society, the index shows its readings on a vertical cylindric surface, which for electric light stations is more convenient than the horizontal scale of the multicellular voltmeters hitherto in use ; but for the measurement of electrostatic capacity the older horizontal scale instrument is as convenient as the new form.

To give a convenient primary electrification for the measurement, a voltaic battery, $\nu \nu^{\prime}$, of about 150 or 200 elements, of each of which the liquid is a drop of water held up by the capillary attraction between a zinc and copper plate about $1 \mathrm{~mm}$. asunder. An ordinary electric machine, or even a stick of rubbed sealing-wax, may, however, be used, but not with the same facility for giving the amount of electrification desired as the voltaic battery.

One end of the voltaic battery is kept joined metallically to a wire, W, dipping in the water in which the cable is submerged, and with the case $C$ of the multicellular, and with the case and piates $\mathrm{A}$ of the Leyden, and with a fixed stud, S, forming part of the operating key to be described later. The other end of the voltaic battery is connected to a flexible insulated wire, FFF, used for giving the primary electrification to the insulated wire $J$ of the cable, and the insulated cells, II, of the multicellular kept metallically connected with it. The insulated plates B of the leyden are connected to a spring, KL, of the operating key referred to above, which, when left to itself, presses down on the metal stud S, and which is very perfectly insulated when lifted from contact with $S$ by a finger applied to the insulating handle $\mathrm{H}$. A second well insulated stud, $\mathrm{S}^{\prime}$, is kept in metallic connection with $J$ and $I$ (the insulated wire of the cable and the insulated cells of the multicellular).

To make a measurement, the flexible wire $\mathrm{F}$ is brought by hand to touch momentarily on a wire connected with the stud $\mathrm{S}^{\prime}$, and immediately after that a reading of the electrometer is taken and watched for a minute or two to test either that there is no sensible loss by imperfect insulation of the cable and the insulated cells of the multicellular, or that the loss is not sufficiently rapid to vitiate the measurement. When the operator is satisfied with this, he records his reading of the electrometer, presses up the handle $\mathrm{H}$ of the key, and so disconnects the plates $\mathrm{B}$ of the leyden from $\mathrm{S}$ and $\mathrm{A}$, and connects them with $S^{\prime}$, J, I. Fifteen or twenty seconds of time suffices to take the thus diminished reading of the multicellular, and the measurement is complete.

The capacity of the cable is then found by the analogy :-As the second reading of the electrometer is to the excess of the

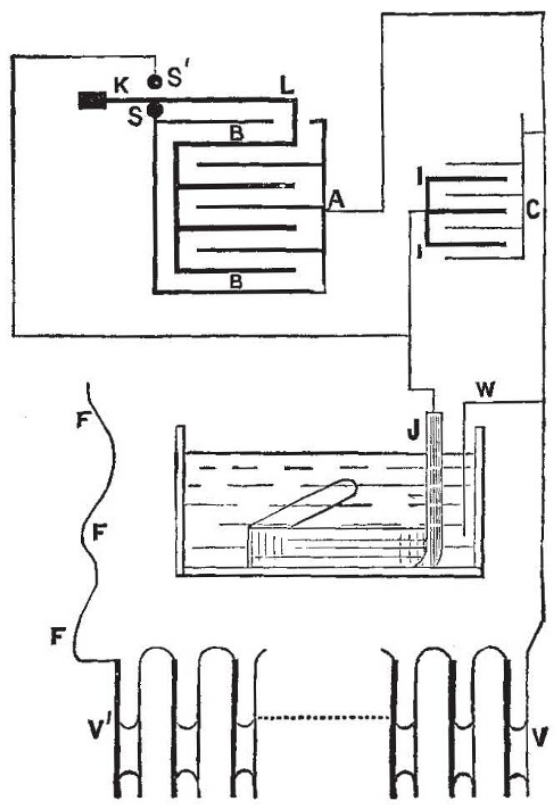

first above the second, so is the capacity of the leyden to the capacity of the cable.

A small correction is readily made with sufficient accuracy for the varying capacity of the electrometer, according to the different positions of the movable plates, corresponding to the different readings, by aid of a table of corrections determined by special measurements for capacity for the purpose on the multicellular.

\section{UNIVERSITY AND EDUCATIONAL INTELLIGENCE.}

CAMBRIDGE.-Prof. Liveing announces a course of lectures in general chemistry, to be given during the Long Vacation by Mr. Fenton, beginning on July 7. Mr. Fenton will also give a series of demonstrations on the chemistry of photography.

At the Congregation on June 16 , seven graduates in arts were admitted to the degree of Doctor in Medicine, and thirty-one to the degrees of Bachelor of Medicine and Bachelor of Surgery. These are the largest numbers hitherto admitted at one time.

Sir R. S. Ball, Lowndean Professor of Geometry and Astronomy, has been elected to a Professorial Fellowship at King's College.

At Christ's College the following awards have been made to students of natural science :-Scholarships: E. K. Jones $\left(6_{50}\right)$ G. A. Anden $(£ 30)$, J. M. Woolley $(£ 30)$, C. F. G. Masterman $(£ 50), H$. Pentecost $(£ 50)$, A. M. Hale $(£ 30)$. Exhibition: A. M. Barraclough $(f 30)$. At Emmanuel College :Scholarship: A. Eichholz (£80). Exhibition: J. C. Muir (£30).

At the annual election of scholars in St. John's College, the following awards in Natural Science have been made :-Foundation Scholarships: W. L. Brown, T. L. Jackson, W. McDougall, S. S. F. Blackman. Exhibitions in Augmentation of Scholarships : Villy, Whipple (First Class Nat. Sci. Tripos, Part II.). Hughes Prize (highest in third year): Villy. Herschel Prize in Astronomy : Pocklington. Hutchinson Studentship for Research in Zoology : E. W. MacBride. 